\title{
Rede própria ou rede credenciada: análise comparativa de custos em uma operadora brasileira de planos de saúde
}

\author{
Marcos Antônio de Souza e Dalva Salvalaio²
}

Como citar Souza MA, Salvalaio D. Rede própria ou rede credenciada: análise comparativa de custos em uma operadora brasileira de planos de saúde. Rev Panam Salud Publica 2010;28(4):305-10.

RESUMO Objetivo. Analisar os custos da rede própria de uma operadora brasileira de planos de saúde comparativamente com o preço cobrado pela rede credenciada, visando a identificar se a opção estratégica da operadora em ter rede própria representou vantagem econômica.

Métodos. Este estudo exploratório foi desenvolvido mediante pesquisa nos relatórios gerenciais da empresa. O custo da rede própria foi calculado com base nas consultas médicas e odontológicas e nos exames diagnósticos e laboratoriais realizados em uma unidade representativa da operadora. Os custos com terceirizados foram obtidos da tabela de preços praticados pela rede credenciada para os mesmos serviços analisados na rede própria. Para a quantificação dos custos foi utilizado o método de custeio pleno. Os custos são apresentados em termos absolutos (em $R \$$ ) e em percentuais dos custos próprios em relação aos da rede credenciada.

Resultados. De modo geral há vantagem para a operadora em utilizar sua própria rede. Isso é verdadeiro para consultas médicas, odontológicas e exames. São exceções as consultas nas especialidades de pediatria e medicina do trabalho e os exames de raio-X.

Conclusões. A opção da operadora pela verticalização do serviço apresenta vantagens econômicas para a empresa em relação à terceirização.

Palavras-chave Seguro saúde; planos de pré-pagamento em saúde; custos e análise de custo; pesquisa sobre serviços de saúde; economia e organizações de saúde; Brasil.

Os estudos relacionados aos serviços de saúde no Brasil, independentemente do seu foco de abordagem e da sua delimitação, têm como aspecto essencial a discussão do sistema de saúde governamental. Esse entendimento decorre do fato de a Constituição Federal de 1988 definir como pública a responsabilidade pela saúde da população (1). É nesse

\footnotetext{
1 Universidade do Vale do Rio dos Sinos (UNISINOS), Curso de Mestrado em Ciências Contábeis. E-mail: marcosas@unisinos.br

2 Faculdade Instituição Evangélica de Novo Hamburgo (Faculdade IENH), Curso de Administração, Novo Hamburgo (RS), Brasil.
}

contexto que surgiu o Sistema Único de Saúde (SUS), para atender a demanda da saúde pública em obediência à determinação constitucional (2).

A implantação do SUS teve como principal justificativa a necessidade de melhorar a oferta de serviços públicos de saúde, contribuindo com avanços qualitativos nos indicadores e nas condições de acesso à saúde (3). Entretanto, o desempenho do SUS tem sido objeto de críticas $(2,4)$, incentivando a procura por planos de saúde ofertados pela iniciativa privada. A expansão dos planos de saúde no Brasil deveu-se à acentuada insatisfação da classe média com a quali- dade dos serviços oferecidos pelo SUS nos atributos conforto, presteza e atendimento personalizado, entre outros (5).

Em 2000, com a criação da Agência Nacional de Saúde Suplementar (ANS), houve mudanças importantes no mercado de planos privados de saúde. Antes da criação da agência, a livre definição da cobertura assistencial permitia à operadora excluir determinados eventos do plano de saúde, como, por exemplo, os exames de alto custo (tomografia e ressonância magnética), ou até mesmo estabelecer o prazo máximo de diárias hospitalares por ano. Além disso, a operadora podia selecionar os clientes aos 
quais venderia planos de saúde. Com essa seleção, a operadora não comercializava planos para idosos ou para portadores de doenças graves como câncer ou Aids. A operadora podia ainda romper contratos de forma unilateral. Assim, se o plano fosse deficitário, a operadora podia processar o seu cancelamento à revelia da vontade do segurado. A carência, período pago pelo beneficiário do plano de saúde sem, entretanto, ter direito de usufruí-lo, também era de livre definição pela operadora. Com a regulamentação, a ANS determinou os prazos máximos de carência para cada tipo de atendimento (6) e impossibilitou, por exemplo, a seleção de clientes e a limitação de coberturas (7).

Essa regulamentação provocou forte impacto econômico e financeiro nas empresas de saúde suplementar, apesar da tendência de expansão do mercado. A causa principal foi a exigência de constituição de garantias financeiras, visando a assegurar a continuidade dos atendimentos. Dados referentes ao período de 2002 a 2008 (8) mostram uma redução de 478 operadoras no período, uma queda de $27,4 \%$, configurando maior concentração no setor. Por outro lado, houve um incremento de 9,8 milhões de beneficiários $(31,5 \%)$, um aumento de $\mathrm{R} \$ 30$ milhões no faturamento $(20,6 \%)$ e de R\$ 25 milhões nos custos $(23,9 \%)$, com redução na margem por segurado de 20,9\% (em 2002) para 19,7\% (em 2008). Além disso, com base no faturamento e no custo per capita, identifica-se que a taxa média de sinistralidade situa-se em $80 \%$, ou seja, para cada $\mathrm{R} \$ 1,00$ recebido, a operadora paga $\mathrm{R} \$ 0,80$ em benefícios para o usuário.

Em função dessas mudanças, algumas operadoras decidiram mudar sua estratégia de atuação, verticalizando suas estruturas, ou seja, criando uma rede própria de atendimento (consultórios, prontoatendimentos, laboratórios, raios- $X$, hospitais). Com a rede própria, as operadoras passaram a ter autonomia e maior controle sobre os custos assistenciais, o que ocorre de forma mais limitada quando os serviços são realizados por uma rede credenciada. Nesse cenário, as informações de custo passam a ser estratégicas, dada a sua relevância para a sustentabilidade dos negócios. De fato, a regulação oficial de mercado e as limitações para correção de preços tornaram a gestão eficiente e eficaz dos custos um pré-requisito à continuidade das operações.
Dentro do contexto apresentado, o objetivo deste estudo é descrever a mensuração e a análise comparativa de custos da rede própria de uma operadora brasileira de planos de saúde em relação ao preço cobrado pela rede credenciada. Entende-se que essa análise comparativa possibilita a identificação de possíveis áreas de desperdício e gera conhecimento básico para uma gestão que efetivamente agregue valor à organização.

\section{MATERIAIS E MÉTODO}

Esta pesquisa de abordagem quantitativa compara os custos da rede própria de uma operadora de saúde com os custos da rede credenciada utilizada pela mesma operadora. Os custos são apresentados em termos absolutos (em R\$) e em percentuais dos custos próprios em relação aos da rede credenciada. A realização deste estudo de caso exploratório envolveu observação, levantamento documental e entrevistas não padronizadas com pessoas que tiveram experiência com o tema pesquisado $(9,10)$. Uma versão mais detalhada do trabalho, enfatizando os aspectos contábeis, foi publicada anteriormente (11).

A operadora de planos de saúde objeto de estudo é uma empresa de medicina de grupo que desenvolve suas atividades em Porto Alegre e na região da Grande Porto Alegre, Estado do Rio Grande do Sul. Como operadora regional, emprega 340 funcionários e detém $4,13 \%$ do mercado, compreendendo 43000 beneficiários. Cerca de $70 \%$ da sua carteira referese a planos coletivos de atendimento ambulatorial e odontológico. O faturamento em 2006 atingiu R\$ 20 milhões, um crescimento de $11 \%$ em relação a 2005. A unidade em análise é uma das oito filiais que a operadora tem estabelecidas na região. Essa unidade atua com 60 funcionários e suas atividades correspondem a $24 \%$ do total do grupo.

A operadora estudada foi selecionada pelo critério de acessibilidade à documentação e aos dados de pesquisa. A realização da pesquisa foi precedida de reunião com diretores, ocasião em que se expôs a abrangência, profundidade e objetivos do estudo. O trabalho foi iniciado após aprovação pela diretoria, e foi acompanhado pelo contador da empresa, encarregado de fornecer os dados necessário. Durante essa fase também ocorreram reuniões com a diretoria para expor o andamento do estudo. $\mathrm{O}$ mesmo ocorreu ao final do estudo, ocasião em que foi aprovada a divulgação dos dados, sendo solicitado apenas o sigilo quanto ao nome e à localização exata da operadora.

Para analisar a concorrência, buscouse informação sobre o número de operadoras registradas na área de atuação da operadora objeto deste estudo. Foram identificadas na mesma região 25 operadoras de medicina de grupo (incluindose aqui o objeto deste estudo), com 540016 beneficiários, e quatro cooperativas de saúde, com 524198 beneficiários. Não se incluem aí as operadoras administradoras (que fazem apenas a administração, e não a execução dos atendimentos que estão a cargo das operadoras) nem as operadoras de autogestão (exclusiva aos funcionários de uma empresa específica). A operadora estudada possui uma carteira de 43000 beneficiários, correspondente a $4,2 \%$ do mercado da região.

Para fazer o comparativo entre a rede própria e a credenciada, primeiramente foi necessário apurar o custo dos atendimentos na rede própria. Como a operadora tem diversas unidades próprias de atendimento, optou-se pela descentralização para cálculo de custos, tomandose como base uma das unidades de atendimento situada na região da Grande Porto Alegre. A unidade selecionada apresenta a melhor estrutura física da operadora, é a única unidade que atende 24 horas por dia e dispõe dos principais serviços que a operadora oferece em sua rede própria. Seu faturamento representa $24 \%$ do total da operadora, com $20 \%$ da carteira de beneficiários. A filial dispõe de 60 funcionários, representando 18\% do quadro total da operadora.

Para o custeio dos atendimentos foram selecionados os serviços de consultas médicas, atendimento odontológico e exames, visto representarem o maior volume de atividades da unidade. $\mathrm{O}$ sistema de custos foi elaborado em planilha eletrônica no programa MS-Excel. Os dados de custo, número de atendimentos e agenda de atendimentos da unidade foram coletados dos relatórios contábeis e gerenciais emitidos pelo sistema operacional (Enterprise Resource Planning, ERP) da operadora. Esses dados refletem o primeiro trimestre de 2007 e foram coletados entre os meses de maio e junho daquele ano. $\mathrm{O}$ tratamento quantitativo dos dados (seleção, análise e tabulação) foi realizado no período de julho a setembro do referido ano. 
Utilizou-se o método de custeio pleno para cálculo do custo dos atendimentos. Neste método considera-se a totalidade dos gastos gerados na unidade, independentemente de serem classificados como custos ou despesas. O método de custeio pleno é mais abrangente do que o método de custeio por absorção (que não considera as despesas) e do que o método de custeio variável (que não considera os custos e despesas fixos no custo dos produtos). Assim, a opção pelo custeio pleno deu-se pela necessidade de comparação dos custos internos com o preço do mesmo serviço ofertado pela rede credenciada. Portanto, o custeio pleno, dadas as suas características, viabiliza a análise da vantagem ou desvantagem de manter um serviço próprio em relação ao atendimento que pode ser feito a um custo maior ou menor na rede credenciada.

Nesse sentido, no processo de custeio dos atendimentos levou-se em consideração os custos diretos (basicamente salários mais encargos dos profissionais atendentes e materiais diretos), acrescidos de parcela correspondente aos custos indiretos (salários e encargos de pessoal de apoio, aluguéis, depreciação, energia elétrica, manutenção, seguros, vigilância, etc.) e das despesas operacionais (administração e comercial), apropriados com base nos diversos critérios de rateio que melhor representam o consumo dos recursos pelos serviços prestados.

Utilizou-se como objeto de custeio o tipo de atendimento realizado na rede própria, ou seja, consultas, exames e odontologia, conforme apresentado na tabela 1. A classificação dos custos e despesas como diretos ou indiretos, fixos ou variáveis, foi feita em relação ao tipo de atendimento, após analisado o fluxo do processo operacional da operadora. Efetuada a classificação, as informações foram apresentadas ao gerente da unidade e ao diretor administrativo para validação. Os custos diretos foram apropriados aos atendimentos e os indiretos rateados de acordo com critérios considerados adequados para este fim.

A rede credenciada pesquisada é formada por serviços contratados pela operadora para atender os beneficiários do plano de saúde. Os contratos definem a forma de prestação do serviço e o montante a ser pago por ele. Os beneficiários precisam de autorização da operadora para atendimento na rede credenciada. A liberação dos atendimentos mediante

TABELA 1. Comparativo de valores das consultas médicas, odontologia e exames na rede própria e na rede credenciada de uma operadora de saúde, Brasil, 2007

\begin{tabular}{|c|c|c|c|c|}
\hline & Custo em $\mathrm{R} \$$ & Preço em $\mathrm{R} \$$ & Variação & Variação \\
\hline Tipo de serviço & Rede própria & Rede credenciada ${ }^{a}$ & $\mathrm{R} \$$ & $\%$ \\
\hline \multicolumn{5}{|l|}{ Consultas médicas } \\
\hline Clínica geral & 18,70 & 22,00 & $(3,30)$ & $-15,0$ \\
\hline Pediatria & 35,59 & 25,00 & 10,59 & 42,4 \\
\hline Ginecologia & 10,78 & 22,00 & $(11,22)$ & $-51,0$ \\
\hline Medicina do trabalho & 20,66 & 20,00 & 0,66 & 3,3 \\
\hline Cirurgia geral & 21,54 & 22,40 & $(0,86)$ & $-3,8$ \\
\hline Ortopedia/traumatologia & 12,74 & 22,00 & $(9,26)$ & $-42,1$ \\
\hline \multicolumn{5}{|l|}{ Odontologia } \\
\hline Dentística & 14,03 & 57,30 & $(43,27)$ & $-75,5$ \\
\hline Endodontia & 18,63 & 86,50 & $(67,87)$ & $-78,5$ \\
\hline Periodontia & 18,52 & 55,10 & $(36,58)$ & $-66,4$ \\
\hline Odontologia saúde coletiva & 12,53 & 29,16 & $(16,63)$ & $-57,0$ \\
\hline Bucomaxilofacial & 22,58 & 46,66 & $(24,08)$ & $-51,6$ \\
\hline Técnico higiene dental & 19,83 & 32,40 & $(12,57)$ & $-38,8$ \\
\hline Odontopediatria & 28,07 & 39,08 & $(11,01)$ & $-28,2$ \\
\hline \multicolumn{5}{|l|}{ Exames } \\
\hline Raios-X & 13,92 & 11,96 & 1,96 & 16,4 \\
\hline Ecografia & 23,74 & 42,08 & $(18,34)$ & $-43,6$ \\
\hline Mamografia & 21,43 & 35,00 & $(13,57)$ & $-38,8$ \\
\hline Eletrocardiograma & 9,36 & 16,20 & $(6,84)$ & $-42,2$ \\
\hline Colonoscopia & 228,55 & 262,79 & $(34,24)$ & $-13,0$ \\
\hline Endoscopia & 62,36 & 284,17 & $(221,81)$ & $-78,1$ \\
\hline Audiometria & 4,76 & 6,00 & $(1,24)$ & $-20,7$ \\
\hline Eletroencefalograma & 27,37 & 37,80 & $(10,43)$ & $-27,6$ \\
\hline Espirometria & 10,16 & 12,00 & $(1,84)$ & $-15,3$ \\
\hline
\end{tabular}

a Dados obtidos nos contratos de credenciados da operadora.

autorização tem como principal objetivo a prévia conferência da cobertura do plano de saúde.

Para realizar o levantamento dos custos com a rede credenciada, solicitou-se à administração da operadora a tabela de preços praticados pela rede credenciada para os mesmos serviços analisados na rede própria, vigente no primeiro semestre do exercício de 2007. Para a seleção dos credenciados utilizaram-se os seguintes critérios: a) foram selecionados os credenciados que ofereciam os serviços disponíveis na rede própria; b) optou-se pelos credenciados localizados na mesma cidade em que é localizada a rede própria-quando o serviço não estava disponível na mesma cidade, optou-se por credenciados da cidade mais próxima; e c) havendo mais de um credenciado que prestasse o mesmo serviço na cidade, optou-se pelo credenciado que oferecia a tabela de menor valor. Como a operadora não contrata rede credenciada para a realização de atendimentos odontológicos, para fazer o comparativo da odontologia foi utilizada a tabela de preços que a Odontoprev, a principal operadora de planos odontológicos do Brasil, repassa aos odontologistas em seu convênio para os serviços constantes da tabela 1 .

\section{RESULTADOS}

A tabela 1 compara o valor das consultas médicas, serviços odontológicos e exames na rede credenciada com o custo apurado na rede própria. Como mostra a tabela, o custo na rede própria da operadora apresenta vantagem em comparação com a rede credenciada nas especialidades de clínica geral, ginecologia, cirurgia geral e ortopedia/traumatologia. Apenas na especialidade de pediatria e medicina do trabalho os custos da rede credenciada foram menores. Na especialidade de pediatria, o custo na rede própria ficou relativamente alto por haver grande ociosidade de agenda no período analisado. Se todos os horários disponíveis da pediatria na rede própria estivessem ocupados, o custo seria de $\mathrm{R} \$ 14,82$, menor do que o praticado pela rede credenciada, que era de $R \$ 25,00$. Outra especialidade que apresenta um custo muito semelhante ao da rede credenciada é a medicina do trabalho, com uma diferença de $\mathrm{R} \$ 0,66$ por consulta realizada. Como essa é uma especialidade que não tem plantão, a redução da carga horária de atendimento poderia melhorar seu desempenho.

Dado que a operadora não utiliza rede credenciada para os serviços odontológicos, utilizou-se, para fins de análise com- 
parativa sobre a eficiência da operadora analisada, o preço cobrado pela principal operadora de planos odontológicos do Brasil, a Odontoprev. Para esses serviços, a tabela 1 mostra expressiva vantagem da rede própria em todos os tipos de atendimento. $\mathrm{Na}$ endodontia foi identificada a diferença mais significativa para a operadora $(78,5 \%)$, sendo apurado, na rede própria, um custo de $\mathrm{R} \$ 18,63$, e na rede credenciada, um preço de $\mathrm{R} \$ 86,50$.

Sobre os exames, é necessário esclarecer que o preço da ecografia e do raio- $X$ nos serviços credenciados variam por tipo de local examinado, ou seja, o raio- $X$ da perna tem um preço, o da coluna tem outro e o do braço um terceiro preço. $\mathrm{Na}$ rede própria, não há diferença de preço, porque a incidência de gastos é a mesma. Assim, utilizou-se uma média de preços dos exames cobrados pelo credenciado selecionado.

Com a análise da tabela 1 pode-se observar que os exames que apresentam vantagem em custo são ecografia, mamografia, eletrocardiograma, colonoscopia, endoscopia, audiometria, eletroencefalograma e espirometria. Apenas o exame de raio- $X$ apresentou custo maior $(16,4 \%)$ para a operadora na rede própria em comparação com a rede credenciada. A diferença mais relevante nos exames ocorre na endoscopia, que apresenta na rede própria um custo unitário de $\mathrm{R} \$ 62,36$, enquanto na rede credenciada, o custo desse exame é de $\mathrm{R} \$ 284,17$.

\section{DISCUSSÃO}

O preço cobrado pelos planos de saúde, conforme regulamentado pela ANS, é apurado através de cálculos atuariais, considerando os custos históricos de atendimento dos beneficiários, ou as estimativas de utilização dos planos. O modo de acesso dos beneficiários aos serviços, a critério da operadora, pode ser de livre escolha (o beneficiário escolhe o prestador de serviços e paga pelo serviço, sendo posteriormente ressarcido pela operadora), escolha dirigida (o beneficiário é atendido, sem pagamento adicional, na rede própria ou na rede credenciada da operadora) ou direcionamento (o beneficiário é encaminhado diretamente a um prestador de serviços específico) (12). A operadora tem ainda a liberdade de definir como vai disponibilizar o serviço de saúde a seus clientes. Algumas operadoras têm rede própria de atendimento, ou seja, em sua estrutura oferecem médicos, centros de diagnóstico e até mesmo hospitais. Outras operadoras trabalham apenas com rede credenciada, tendo todos os serviços do plano de saúde terceirizados. Há também a possibilidade de oferecer o serviço tanto na rede própria como na rede credenciada. Tem-se assim uma verticalização total ou parcial (13).

A operadora que possui rede própria atende grande parte de seus beneficiários nesse local, mas, como os planos de saúde têm cobertura ampla, nem sempre todos os serviços de saúde necessários ao atendimento dos beneficiários estão disponíveis na rede própria. Por esse motivo, as operadoras utilizam-se também da rede credenciada. Entretanto, o uso de rede credenciada exige uma duplicação das atividades de gestão: além dos cuidados para que sejam executados apenas os atendimentos realmente necessários, é preciso lembrar que a responsabilidade final pelo serviço perante o usuário é da operadora, e não da rede credenciada. Esse controle de qualidade torna-se assim uma atividade adicional na estrutura da operadora do plano de saúde.

É reconhecido que o controle de custos tornou-se fundamental para o mercado de planos de saúde. Isso ocorre de forma mais acentuada desde a regulamentação oficial, tendo em vista o aumento dos gastos com a ampliação obrigatória da cobertura (14). Além disso, há o controle dos reajustes dos planos de pessoa física, fixado pelo governo federal. Observa-se uma tendência das operadoras em investir na rede própria (15).

Deve-se reconhecer que há interesses antagônicos na relação entre a operadora e a sua rede credenciada. Para a operadora, quanto menor for a utilização dos beneficiários, maior a sua rentabilidade e, para o prestador de serviços da rede credenciada, quanto maior for a utilização do usuário do plano de saúde, maior o seu retorno (13). Esse é um dos principais motivos para a verticalização dos serviços por parte das operadoras. É importante destacar ainda que ter uma rede própria não confere à operadora apenas o controle da utilização dos beneficiários, mas também oferece maior poder de negociação junto à rede credenciada, pois a operadora tem uma alternativa para atendimento.

O sistema de custos faz parte do sistema de gestão da empresa, devendo estar alinhado a ele para que as infor- mações geradas viabilizem resultados úteis (16). Entretanto, há um complicador no gerenciamento de custos das operadoras, pois neste caso há um regime onde as receitas são fixas e pré-determinadas, enquanto os custos com assistência à saúde são, pelo menos em parte, variáveis em função dos atendimentos. Assim, a operadora recebe mensalmente uma receita, conhecida desde que comercializou o plano, mas desconhece o custo que o plano pode gerar, dificultando uma previsão da margem a ser obtida com o plano (17).

A esse respeito, e no contexto da gestão de custos, estudos alertam que minimizar custos em serviços de saúde pode levar a resultados contraproducentes. A economia de custos deve advir de reais eficiências na prestação de serviços, e não da restrição de tratamentos ou da redução da sua qualidade (18). Além disso, deve-se compreender que o aumento dos custos em instituições de saúde está relacionado com os avanços da medicina e da tecnologia colocada à disposição dos médicos, que passam a gerar um custo mais elevado para o mesmo tipo de serviço (19) e isso é particularmente relevante com relação aos exames de diagnóstico.

De fato, os exames sofisticados impactam significativamente na ocorrência de custos da operadora. A livre escolha de médicos e prestadores de serviços credenciados pelo beneficiário, na ótica do cliente, é um sinal da qualidade do plano adquirido, mas, para as operadoras, representa potencial de maior custo, pois o beneficiário pode consultar com vários médicos e repetir os mesmos exames sem nenhum tipo de controle (20).

A par dessas questões específicas do segmento de serviços de saúde, deve-se observar que a gestão de custos visa a melhoria da gestão, mas não é suficiente para isoladamente manter a empresa competitiva (21). Assim, a integração de custos com a estratégia passa a ser relevante para uma análise mais profunda sobre a determinação do rumo estratégico da empresa. A análise aqui exposta sobre os custos da rede própria, de forma comparativa com a rede credenciada, gera dados que podem auxiliar a tomada de decisões voltadas às necessidades operacionais e estratégicas, impactando assim na forma de administração das operadoras.

Analisando os dados do comparativo com a rede credenciada, observa-se que, 
no caso da operadora analisada, a verticalização é uma estratégia favorável. Observa-se que os resultados apurados não podem ser extrapolados para outros estados do país, tendo em vista que o Brasil é composto por realidades econômicas e sociais muito diferentes, com acentuados reflexos nos níveis de preços e custos. Apesar dessa limitação, há neste estudo a geração de bases, inclusive metodológicas, que podem servir de parâmetros para a realização de outros estudos dessa natureza.

A variação do custo entre a rede própria e a rede credenciada pode ser consequência de diversos fatores. Por exemplo, a rede própria tem em sua estrutura diversos serviços que dividem gastos de área física, energia e pessoal, otimizando recursos para a operadora. Já o serviço credenciado normalmente oferece apenas um tipo de serviço, tendo um custo mais alto para a sua manutenção. A rede credenciada deve considerar, também, uma margem de lucro embutida no seu preço, o que não é interessante para a rede própria da operadora, já que a receita é proveniente da mensalidade de planos de saúde, e quanto menor for o custo do atendimento desses beneficiários, melhor o resultado da operadora. Além disso, a rede credenciada precisa repassar o custo da sua ociosidade no preço, já que a sua estrutura de atendimento tem uma dimensão básica fixa em termos de capacidade produtiva e de custos. Na rede própria, o serviço é dimensionado de acordo com a necessidade da operadora e, quando há ociosidade, a mesma passa a ser absorvida pela operadora, principalmente no que tange às necessidades técnicas. Um exemplo concreto é o fato de que, no presente estudo de caso, a opção da operadora pelo não credenciamento de serviços terceirizados para atendimento odontológico foi uma decisão acertada, pois todos os tipos de atendimento odontológico observados tinham custo maior na rede credenciada.

Tendo em vista ser de responsabilidade pública governamental cuidar da saúde da população, conforme citado inicialmente, é reconhecida a necessidade de a iniciativa privada estar presente de forma complementar. A dificuldade do Estado de cumprir o seu encargo constitucional é reconhecida tacitamente pela criação da Agência Nacional de Saúde Suplementar. Nesse sentido, cabe à ANS cuidar, também, da saúde financeira das operadoras de planos de saúde, intervindo se necessário. Nesse sentido, é importante a contribuição deste estudo, ao analisar o formato operacional que melhor resultado econômico propicia às operadoras, um requisito para a sua continuidade sustentável e para a complementação dos serviços públicos de saúde, razão da sua criação.

O que se tem como premissa para este estudo é que a operadora de saúde, a par do seu compromisso social, é uma entidade econômica, assim como o é o Estado e, como tal, tem que ser eficiente e eficaz em suas atividades, o que implica no melhor uso possível dos recursos disponíveis. Essa viabilidade econômica pode possibilitar uma contribuição mais ativa para a melhoria da saúde pública, por exemplo, pelo financiamento de atividades governamentais, embora ainda seja necessário delinear a forma como tal financiamento pode se viabilizar. Um mecanismo fiscal diferenciado pode ser uma alternativa a ser avaliada.

A pesquisa bibliográfica realizada por ocasião do presente trabalho identificou um expressivo crescimento nos estudos sobre os custos na área médica. Entretanto, são poucos os estudos que abordam os custos dos planos de saúde e sua gestão pelas operadoras. Além disso, em geral não há um detalhamento dos custos no nível analítico dos procedimentos, como no presente estudo: as abordagens costumam ser genéricas, com discussão de métodos de custos e suas necessidades, sem foco na mensuração e no custeio $(1,22-24)$.

Fica, portanto, clara a necessidade de outros estudos dessa natureza, principalmente se considerada a expressiva representatividade das empresas de planos de saúde. Isso é mais relevante quando sabe-se da grande variabilidade econômica, estrutural e cultural no âmbito regional e estadual que caracteriza um país das dimensões continentais como o Brasil. Por fim, essa falta de outros estudos com o mesmo enfoque tratado no presente artigo traz como consequência a limitação da análise, prejudicando uma visão e entendimento mais abrangentes dos resultados aqui encontrados.

\section{Considerações finais}

Este estudo teve como objetivo a análise comparativa entre o custo apurado na rede própria de uma operadora brasi- leira de plano de saúde e o preço do mesmo serviço na rede credenciada. Com tal estudo buscou-se identificar se a opção da operadora pela verticalização do serviço foi mais vantajosa para a empresa.

Em geral, a opção pela rede própria é vantajosa. Observou-se que as consultas médicas das especialidades de pediatria e medicina do trabalho apresentam valor menor na rede credenciada que na rede própria. Na odontologia, todos os tipos de atendimento foram mais vantajosos na rede própria que na rede credenciada. Nos exames, exceto o raio- $X$, que apresenta custo mais elevado na rede própria que na rede credenciada, os demais exames têm vantagem em custo para a operadora na rede própria. Essas informações devem ser analisadas pelos gestores da empresa para que seja revertida a situação daqueles serviços que se mostrarem deficitários, mediante a adoção de ações no sentido de reduzir o custo, apesar da lucratividade global que a operadora historicamente tem atingido.

O sistema de custos da rede própria da operadora é um instrumento gerencial importante no mercado em que atua. Se avaliado em conjunto com os preços praticados pela rede credenciada, esse instrumento define a melhor decisão estratégica-se voltada para a verticalização ou para a terceirização do serviço-e possibilita a análise da verticalização de outros serviços para a operadora obter melhores resultados.

Como sugestão para outros estudos no segmento da prestação de serviços de saúde, e com base nos aspectos observados durante o desenvolvimento desta pesquisa, coloca-se uma agenda destinada a superar o baixo número de trabalhos analíticos de mensuração e custeio. Além disso, cabe ainda investigar quais os instrumentos e práticas de gestão de custos, no âmbito gerencial e estratégico, que mais têm se mostrado eficazes nas operadoras de plano de saúde nas mais diversas regiões de estados e municípios brasileiros. Acredita-se que essas abordagens poderão trazer maior divulgação e conscientização a respeito da gestão de custos nessa área de prestação de serviços de saúde. Isso parece ser particularmente apropriado dadas as características de um mercado sob forte regulamentação governamental e de acirrada disputa entre os seus principais agentes. 


\section{REFERÊNCIAS}

1. Silva LM, Souza LE, Cerdeira AJ, Pinto, CM, Oliveira RS. Algumas características do setor privado de saúde de Salvador, Bahia, Brasil. Cad Saude Publica. 1997;13(4):701-9.

2. Szymczak VL, Pires AJ. O sistema único de saúde (SUS) no contexto da seguridade social: a diferença entre a realidade no cotidiano social e as determinações legais vigentes. Rev Eletronica Lato Sensu. 2006;2(1):1-22.

3. Medici AC. História e políticas de saúde no Brasil: um balanço do processo de descentralização [tese]. São Paulo: Universidade de São Paulo; 1999.

4. Backes DS, Koerich MS, Rodrigues AC, Drago LC, Klock P, Erdmann AL. O que os usuários pensam e falam do Sistema Único de Saúde? Uma análise dos significados à luz da carta dos direitos dos usuários. Cien Saude Colet. 2009;14(3):903-10.

5. Ribeiro JM. Restrições de informações, custos de transação e ambiente regulatório em saúde suplementar. 2004. Disponível em: www.ans. gov.br/portal/upload/biblioteca/TT_OS_ JoseMRibeiroSaudeSuplementarEFalhasDe Inform.pdf. Acessado em 31 de julho de 2009.

6. Brasil, Ministério da Saúde, Agência Nacional de Saúde Suplementar. Resolução normativa $\mathrm{n}^{\circ} 27$, de 1 de abril de 2003. Disponível em: www.ans.gov.br/portal/site/legislacao/ legislacao_integra.asp?id=384\&id_original $=0 /$. Acessado em setembro de 2010.

7. Ocké-Reis CO. Challenges in the regulation of Brazilian private health insurance. Public Finance Manag. 2005;5(4):597-624.

8. Brasil, Ministério da Saúde, Agência Nacional de Saúde Suplementar. Caderno de Informações da Saúde Suplementar. Edição março 2009. Disponível em: www.ans.gov.br/portal/ upload/informacoesss/caderno_informaca 03_2009.pdf. Acessado em 21 de maio de 2009.
9. Gil AC. Métodos e técnicas de pesquisa social. $5^{\mathrm{a}}$ ed. São Paulo: Atlas; 1999.

10. Roesch SMA. Projetos de estágio e de pesquisa em administração. $3^{a}$ ed. São Paulo: Atlas; 2006.

11. Salvalaio D. Custos na rede própria de operadora de planos de saúde: um estudo no âmbito da gestão descentralizada [dissertação de mestrado]. São Leopoldo, RS: UNISINOS; 2008. Disponível em: http://bdtd.unisinos. $\mathrm{br} /$ tde_busca/arquivo.php? codArquivo=739. Acessado em outubro de 2010.

12. Saito R, Schiozer RF, Saito CC. Situação financeira e satisfação do consumidor das operadoras de saúde suplementar no Brasil [CDROM]. Em: Anais do XXXI Encontro da Associação Nacional de Pós-Graduação e Pesquisa em Administração; 2007; Rio de Janeiro: ANPAD; 2007.

13. Alves SL. Uma análise econômica da verticalização no mercado de saúde suplementar. Rev Eletronica Administraçao Hospitalar. 2006;2(6 set/dez):1-11.

14. Montone J. Evolução e desafios da regulação do setor de saúde suplementar. Em: Brasil, Ministério da Saúde, Agência Nacional de Saúde Suplementar. Documentos técnicos de apoio ao Fórum de Saúde Suplementar em 2003. Rio de Janeiro: ANS; 2003. Pp. 9-48.

15. Araújo W. Mercado atraente. Correio Brasiliense 200630 de outubro. Disponível em: www.fbh.com.br/index.php?ID_MATERIA= $5577 \&$ busca $=1$. Acessado em setembro de 2010.

16. Bornia AC. Análise gerencial de custos: aplicação em empresas modernas. Porto Alegre: Bookman; 2002.

17. Miranda CR. Gerenciamento de custos em planos de assistência à saúde. Projeto ANS/ PNUD. 2003. Disponível em: www.ans.gov.
br/portal/upload/biblioteca/TT_AS_20 ClaudioMiranda_GerenciamentodeCusto.pdf. Acessado em 29 de junho de 2009.

18. Porter ME, Teisberg EO. Redefining competition in health care. Harv Bus Rev. 2004;82(6): 65-79, 136.

19. Capettini R, Chow CW, McNamee AH. On the need and opportunities for improving costing and cost management in healthcare organization. Managerial Finance. 1998;24(1): 46-59.

20. Marinho F, Mac-Allister M. Distribuição espacial dos serviços de saúde para as organizações prestadoras privadas [CD-ROM]. Em: Anais do XXIX Encontro da Associação Nacional de Pós-Graduação e Pesquisa em Administração; 2005 17-21 set; Brasília: ANPAD; 2005.

21. Bacic MJ. Escopo da gestão estratégica de custos em face das noções de competitividade e de estratégia empresarial [CD-ROM]. Em: Anais do I Congresso Brasileiro de Gestão Estratégica de Custos; 1994 20-23 nov; São Leopoldo, Brasil.

22. Reis COO. O Estado e os planos de saúde no Brasil. Rev Serviço Publico. 2000;51(1):123-147.

23. Pinto LF, Soranz DR. Planos privados de assistência à saúde: cobertura populacional no Brasil. Cien Saude Colet. 2004;9(1):85-98.

24. Alves SL. Saúde suplementar: evidências de seleção adversa após regulação [CD-ROM]. Em: Anais do XXXV Encontro Nacional de Economia; 2007 4-7 dez; Recife: ANPEC; 2007.

Manuscrito recebido em 13 de outubro de 2009. Aceito em versão revisada em 10 de junho de 2010.
ABSTRACT

\section{Self-owned versus accredited network: comparative cost analysis in a Brazilian health insurance provider}

Key words
Objective. To analyze the cost of a self-owned network maintained by a Brazilian health insurance provider as compared to the price charged by accredited service providers, so as to identify whether or not the self-owned network is economically advantageous.

Methods. For this exploratory study, the company's management reports were reviewed. The cost associated with the self-owned network was calculated based on medical and dental office visits and diagnostic/laboratory tests performed at one of the company's most representative facilities. The costs associated with third parties were derived from price tables used by the accredited network for the same services analyzed in the self-owned network. The full-cost method was used for cost quantification. Costs are presented as absolute values (in $\mathrm{R} \$$ ) and percent comparisons between self-owned network costs versus accredited network costs.

Results. Overall, the self-owned network was advantageous for medical and dental consultations as well as diagnostic and laboratory tests. Pediatric and labor medicine consultations and $\mathrm{x}$-rays were less costly in the accredited network.

Conclusions. The choice of verticalization has economic advantages for the health care insurance operator in comparison with services provided by third parties.

Insurance, health; prepaid health plans; costs and cost analysis; health services research; health care economics and organizations; Brazil. 